\title{
Compatible Mappings and Some Common Fixed Point Results
}

\author{
T. Som ${ }^{1, *}$, A. Choudhury ${ }^{2}$ \\ ${ }^{1}$ Department of Applied Mathematics, Institute of Technology, Banaras Hindu University, Varanasi, 221005, India \\ ${ }^{2}$ Department of Mathematics and Statistics, Haflong Govt. College, Haflong, Dima Hasao, Assam, 788819, India
}

\begin{abstract}
The present paper deals with some common fixed point results on a metric space for compatible mappings satisfying a more general inequality condition. The results obtained generalize the fixed point results of Mukherjee (1981) and Som (1985) and many others in turn.
\end{abstract}

Keywords Compatible Mappings, Metric Space, Fixed Point

\section{Introduction}

The idea of common fixed point was initially given by Jungck[3], where the mappings considered were commuting. There after many generalizations of this common fixed point result were obtained by several mathematicians viz., Hadzic[2], Meade and Singh[11], Pathak[7], Yeh[13] etc. The commutativity condition of mappings was further replaced by a weaker type of notion viz., weakly commuting mappings as introduced by Sessa[8]. Several common fixed point theorems have been proved for such mappings by many authors viz., Sessa et. al.[9], Fisher and Sessa[1] and others. The notion of weak commutativity has been further weakened by the notion of compatible mappings, introduced by Jungck[4] which gave a new direction towards more comprehensive results in the context of common fixed point in metric fixed point theory. In this paper we obtain some common fixed point results for compatible mappings satisfying more general inequality condition which mainly generalize the results of Mukherjee[6], Som[10] and others in turn.

\subsection{Definition I (Sessa[8]) :}

Two self mappings $\mathrm{A}$ and $\mathrm{S}$ of a metric space $(\mathrm{X}, \mathrm{d})$ are called weakly commuting if

$$
\mathrm{d}(\mathrm{ASx}, \mathrm{SAx}) \leq \mathrm{d}(\mathrm{Ax}, \mathrm{Sx}) \text { for every } \mathrm{x} \in \mathrm{X} .
$$

\subsection{Definition II (Jungck[4]) :}

Let $f$ and $g$ be two self maps on a metric space $(X, d)$. $f$ and $\mathrm{g}$ are called compatible if

$$
\lim _{n \rightarrow \infty} d\left(f g x_{n}, g f x_{n}\right)=0
$$

* Corresponding author:

tsom.apm@itbhu.ac.in (T. Som)

Published online at http://journal.sapub.org/ajms

Copyright (C) 2011 Scientific \& Academic Publishing. All Rights Reserved whenever $\left\{x_{n}\right\}$ is a sequence such that $\lim _{n \rightarrow \infty} \mathrm{fx}_{\mathrm{n}}=\lim _{\mathrm{n} \rightarrow \infty} \mathrm{gx}_{\mathrm{n}}=\mathrm{t}$ for some $\mathrm{t}$ in $\mathrm{X}$.

The Banach contraction theorem assures fixed point for a mapping which is necessarily continuous. However Taskovic[12] proved the following fixed point result for a self mapping which is not necessarily continuous.

\subsection{Theorem I}

Let $\mathrm{T}$ be a self mapping of a complete metric space $(\mathrm{X}, \mathrm{d})$ satisfying

(1) $\operatorname{ad}(T x, T y)+b d(x, T x)+\operatorname{cd}(y, T y)-\min \{d(T x, y)$, $\mathrm{d}(\mathrm{x}, \mathrm{Ty})\} \leq \mathrm{qd}(\mathrm{x}, \mathrm{y})$

with $\mathrm{a}>\mathrm{q}+1$ and $\mathrm{a}+\mathrm{c}>0$. Then $\mathrm{T}$ has a unique fixed point in X.

Generalizing the above result of Taskovic and that of Mukherjee[6] for common fixed point of two mappings, Som[10] obtained the following results.

\subsection{Theorem II}

Let $\mathrm{T}$ and $\mathrm{S}$ be two self mappings of a complete metric space $(X, d)$ satisfying

(2) $\mathrm{ad}(\mathrm{Tx}, \mathrm{Sy})+\mathrm{bd}(\mathrm{x}, \mathrm{Tx})+\mathrm{cd}(\mathrm{y}, \mathrm{Sy})-\min \{\mathrm{d}(\mathrm{x}, \mathrm{Sy})$,

$$
\mathrm{d}(\mathrm{Tx}, \mathrm{y})\} \leq \mathrm{qd}(\mathrm{x}, \mathrm{y})
$$

for all $\mathrm{x}, \mathrm{y} \in \mathrm{X}$, where $\mathrm{a}, \mathrm{b}, \mathrm{c} \geq 0, \mathrm{q}>0$ with $\mathrm{a}>\mathrm{q}+1$ and $\mathrm{a}+\mathrm{c}>0$. Then $\mathrm{T}$ and $\mathrm{S}$ have a unique common fixed point.

\subsection{Theorem 1.3.}

Let $(\mathrm{X}, \mathrm{d})$ be a complete metric space and $\mathrm{T}$ and $\mathrm{S}$ be a self mappings of $X$ satisfying

(3) ad $(\mathrm{Tx}, \mathrm{Sy})+\mathrm{bd}(\mathrm{x}, \mathrm{Tx})+\mathrm{cd}(\mathrm{y}, \mathrm{Sy})-\min \{\mathrm{d}(\mathrm{x}, \mathrm{Sy})$, $\mathrm{d}(\mathrm{Tx}, \mathrm{y})\} \leq q \max \{\mathrm{d}(\mathrm{x}, \mathrm{y}), \mathrm{d}(\mathrm{x}, \mathrm{Tx}), \mathrm{d}(\mathrm{y}, \mathrm{Sy})$

$$
[\mathrm{d}(\mathrm{x}, \mathrm{Sy})+\mathrm{d}(\mathrm{Tx}, \mathrm{y})] / 2\}
$$

for all $\mathrm{x}, \mathrm{y} \in \mathrm{X}$, where $\mathrm{a}, \mathrm{b}, \mathrm{c} \geq 0, \mathrm{q}>0$ with $\mathrm{a}>\mathrm{q}+1$ and $\mathrm{a}$ $+\mathrm{c}>0$. Then $\mathrm{T}$ and $\mathrm{S}$ have a unique common fixed point.

Unifying the mapping conditions of above theorems we obtain few common fixed point results in the next section. 


\section{Main Results}

Our first theorem extends the result of Som[10] in respect of the mapping condition.

\subsection{Theorem I}

Let $A$ be a self mapping of a complete metric space $(X, d)$ and $\mathrm{S}, \mathrm{T}$ be two continuous self mapping of $\mathrm{X}$ satisfying

(i) the pairs $\{\mathrm{A}, \mathrm{S}\}$ and $\{\mathrm{A}, \mathrm{T}\}$ are compatible with $\mathrm{A}(\mathrm{X}) \subset \mathrm{S}(\mathrm{X}) \cap \mathrm{T}(\mathrm{X})$.

(ii) $\operatorname{ad}(\mathrm{Ax}, \mathrm{Ay})+\mathrm{bd}(\mathrm{Sx}, \mathrm{Ty})+\operatorname{cd}(\mathrm{Sx}, \mathrm{Ax}) \leq \phi\{\mathrm{d}(\mathrm{Sx}, \mathrm{Ty})$, $\mathrm{d}(\mathrm{Sx}, \mathrm{Ax}), \mathrm{d}(\mathrm{Sx}, \mathrm{Ay}), \mathrm{d}(\mathrm{Ty}, \mathrm{Ax}), \mathrm{d}(\mathrm{Ty}, \mathrm{Ay})\}$

where $\mathrm{a}+\mathrm{b}+\mathrm{c}>1$ and $\phi: \mathrm{R}_{+}{ }^{5} \rightarrow \mathrm{R}_{+}$is non-decreasing in each coordinate variable and for any $\mathrm{t}>0, \phi\left(t, t, a_{1} t, a_{2} t, t\right)<t, a_{1}$ $+\mathrm{a}_{2}=3$. Then $\mathrm{A}, \mathrm{S}$ and $\mathrm{T}$ have a unique common fixed point in $\mathrm{X}$.

Proof: Let $\mathrm{x}_{0}$ be any arbitrary point in $\mathrm{X}$. Then $\mathrm{Ax}_{0} \in \mathrm{X}$. Since $A(X) \subset S(X)$, there exists a point $x_{1} \in X$ such that $A x_{0}$ $=\mathrm{Sx}_{1}$. Further since $\mathrm{A}(\mathrm{X}) \subset \mathrm{T}(\mathrm{X})$, there is a point $\mathrm{x}_{2} \in \mathrm{X}$ such that $\mathrm{Ax}_{1}=\mathrm{Tx}_{2}$. In general we have the sequence $\left\{\mathrm{x}_{\mathrm{n}}\right\}$ in $X$ such that

$$
\begin{gathered}
\mathrm{Ax}_{2 \mathrm{n}}=\mathrm{Sx}_{2 \mathrm{n}+1}, \mathrm{Ax}_{2 \mathrm{n}+1}=\mathrm{Tx}_{2 \mathrm{n}+2}, \mathrm{n}=0,1,2, \ldots \\
\text { Let } \mathrm{d}_{\mathrm{n}}=\mathrm{d}\left(\mathrm{Ax}_{\mathrm{n},} \mathrm{Ax}_{\mathrm{n}+1}\right)>0, \mathrm{n}=0,1,2, \ldots .
\end{gathered}
$$

Here we claim that $\left\{d_{n}\right\}$ is a decreasing sequence. Suppose that $d_{2 n}>d_{2 n-1}$ for some $n$. Putting $x=x_{2 n+1}, y=x_{2 n}$ in (ii), we get

$$
\begin{aligned}
& \operatorname{ad}\left(\mathrm{Ax}_{2 \mathrm{n}+1}, \mathrm{Ax}_{2 \mathrm{n}}\right)+\mathrm{bd}\left(\mathrm{Sx}_{2 \mathrm{n}+1}, \mathrm{Tx}_{2 \mathrm{n}}\right)+\mathrm{cd}\left(\mathrm{Sx}_{2 \mathrm{n}+1}, \mathrm{Ax}_{2 \mathrm{n}+1}\right) \\
& \leq \phi\left\{d\left(\mathrm{Sx}_{2 \mathrm{n}+1}, \mathrm{Tx}_{2 \mathrm{n}}\right), \mathrm{d}\left(\mathrm{Sx}_{2 \mathrm{n}+1}, \mathrm{Ax}_{2 \mathrm{n}+1}\right), \mathrm{d}\left(\mathrm{Sx}_{2 \mathrm{n}+1}, \mathrm{Ax}_{2 \mathrm{n}}\right)\right. \text {, } \\
& \left.\mathrm{d}\left(\mathrm{Tx}_{2 \mathrm{n}}, \mathrm{Ax}_{2 \mathrm{n}+1}\right), \mathrm{d}\left(\mathrm{Tx}_{2 \mathrm{n}}, \mathrm{Ax}_{2 \mathrm{n}}\right)\right\} \\
& \text { or, } \operatorname{ad}\left(\mathrm{Ax}_{2 \mathrm{n}+1}, \mathrm{Ax}_{2 \mathrm{n}}\right)+\mathrm{bd}\left(\mathrm{Ax}_{2 \mathrm{n}}, \mathrm{Ax}_{2 \mathrm{n}-1}\right)+\mathrm{cd}\left(\mathrm{Ax}_{2 \mathrm{n}}, \mathrm{Ax}_{2 \mathrm{n}+1}\right) \\
& \leq \phi\left\{d\left(A x_{2 n}, A x_{2 n-1}\right), d\left(A x_{2 n}, A x_{2 n+1}\right), d\left(A x_{2 n}, A x_{2 n}\right),\right. \\
& \left.\mathrm{d}\left(\mathrm{Ax}_{2 \mathrm{n}-1}, \mathrm{Ax}_{2 \mathrm{n}+1}\right), \mathrm{d}\left(\mathrm{Ax}_{2 \mathrm{n}-1}, \mathrm{Ax}_{2 \mathrm{n}}\right)\right\} \\
& \text { or, } \mathrm{ad}_{2 \mathrm{n}}+\mathrm{bd}_{2 \mathrm{n}-1}+\mathrm{cd}_{2 \mathrm{n}} \leq \phi\left\{\mathrm{d}_{2 \mathrm{n}-1}, \mathrm{~d}_{2 \mathrm{n}}, 0, \mathrm{~d}_{2 \mathrm{n}-1}+\mathrm{d}_{2 \mathrm{n}}, \mathrm{d}_{2 \mathrm{n}-1}\right\} \\
& \text { or, }(\mathrm{a}+\mathrm{c}) \mathrm{d}_{2 \mathrm{n}}+\mathrm{bd}_{2 \mathrm{n}-1}<\phi\left\{\mathrm{d}_{2 \mathrm{n}}, \mathrm{d}_{2 \mathrm{n}}, \mathrm{d}_{2 \mathrm{n}}, 2 \mathrm{~d}_{2 \mathrm{n}}, \mathrm{d}_{2 \mathrm{n}}\right\}<\mathrm{d}_{2 \mathrm{n}} \\
& \text { or, } \\
& \mathrm{d}_{2 \mathrm{n}}<\frac{-\mathrm{b}}{\mathrm{a}+\mathrm{c}-1} \mathrm{~d}_{2 \mathrm{n}-1}<\mathrm{d}_{2 \mathrm{n}-1}
\end{aligned}
$$

i.e., a contradiction. Therefore $\left\{\mathrm{d}_{\mathrm{n}}\right\}$ is decreasing sequence of non negative reals, so it converges to zero. Now, we show that $\left\{A x_{n}\right\}$ is a Cauchy sequence. Since $\lim _{n \rightarrow \infty} d_{n}=$ 0 , it is sufficient to show that $\left\{A_{2 n}\right\}$ is a Cauchy sequence. Suppose that it is not so. Then there is an $\varepsilon>0$ and for each even integer $2 \mathrm{k}, \mathrm{k}=0,1,2, \ldots$ there exists even integer $2 \mathrm{n}(\mathrm{k})$ and $2 \mathrm{~m}(\mathrm{k})$ with $2 \mathrm{k}<2 \mathrm{n}(\mathrm{k})<2 \mathrm{~m}(\mathrm{k})$ such that

(iii) $\mathrm{d}\left(\mathrm{Ax}_{2 \mathrm{n}(\mathrm{k})}, \mathrm{Ax}_{2 \mathrm{~m}(\mathrm{k})}\right)>\varepsilon$

Let for each even integer $2 \mathrm{k}, 2 \mathrm{~m}(\mathrm{k})$ be least positive integer exceeding $2 \mathrm{n}(\mathrm{k})$ satisfying (iii),

then

(iv) $\mathrm{d}\left(\mathrm{Ax}_{2 \mathrm{n}(\mathrm{k})}, \mathrm{Ax}_{2 \mathrm{~m}(\mathrm{k})-2)}\right) \varepsilon$ and $\mathrm{d}\left(\mathrm{Ax}_{2 \mathrm{n}(\mathrm{k})}, \mathrm{Ax}_{2 \mathrm{~m}(\mathrm{k})}\right)>\varepsilon$ As such for each even integer $2 \mathrm{k}$, we have

$$
\begin{aligned}
\varepsilon<\mathrm{d}\left(\mathrm{Ax}_{2 \mathrm{n}(\mathrm{k})}, \mathrm{Ax}_{2 \mathrm{~m}(\mathrm{k})}\right) \leq & \mathrm{d}\left(\mathrm{Ax}_{2 \mathrm{n}(\mathrm{k})}, \mathrm{Ax}_{2 \mathrm{~m}(\mathrm{k})-2}\right)+\mathrm{d}_{2 \mathrm{~m}(\mathrm{k})-2} \\
& +\mathrm{d}_{2 \mathrm{~m}(\mathrm{k})-1}
\end{aligned}
$$

So by (iv) and as $\mathrm{k} \rightarrow \infty$, we get

(v) $\lim _{\mathrm{k} \rightarrow \infty} \mathrm{d}\left(\mathrm{Ax}_{2 \mathrm{n}(\mathrm{k})}, \mathrm{Ax}_{2 \mathrm{~m}(\mathrm{k})}\right)=\varepsilon$

Now using (v) in the triangle inequalities

$$
\left|\mathrm{d}\left(\mathrm{Ax}_{2 \mathrm{n}(\mathrm{k})}, A x_{2 \mathrm{~m}(\mathrm{k})-1}\right)-\mathrm{d}\left(\mathrm{Ax}_{2 \mathrm{n}(\mathrm{k})}, A x_{2 \mathrm{~m}(\mathrm{k})}\right)\right| \leq \mathrm{d}_{2 \mathrm{~m}(\mathrm{k})-1}
$$
and $\left|\mathrm{d}\left(\mathrm{Ax}_{2 \mathrm{n}(\mathrm{k})+1}, \mathrm{Ax}_{2 \mathrm{~m}(\mathrm{k})-1}\right)-\mathrm{d}\left(\mathrm{Ax}_{2 \mathrm{n}(\mathrm{k})}, \mathrm{Ax}_{2 \mathrm{~m}(\mathrm{k})}\right)\right|$ $\leq \mathrm{d}_{2 \mathrm{~m}(\mathrm{k})-1}+\mathrm{d}_{2 \mathrm{n}(\mathrm{k})}$.

Taking limit as $\mathrm{k} \rightarrow \infty$, we get $\mathrm{d}\left(\mathrm{Ax}_{2 \mathrm{n}(\mathrm{k})}, \mathrm{Ax}_{2 \mathrm{~m}(\mathrm{k})-1}\right) \rightarrow \varepsilon$ and $\mathrm{d}\left(\mathrm{Ax}_{2 \mathrm{n}(\mathrm{k})+1}, \mathrm{Ax}_{2 \mathrm{~m}(\mathrm{k})-1}\right) \rightarrow \varepsilon$. Then,

$$
\begin{aligned}
& \mathrm{d}\left(\mathrm{Ax}_{2 \mathrm{n}(\mathrm{k})}, \mathrm{Ax}_{2 \mathrm{~m}(\mathrm{k})}\right) \leq \mathrm{d}\left(\mathrm{Ax}_{2 \mathrm{n}(\mathrm{k})}, \mathrm{Ax}_{2 \mathrm{n}(\mathrm{k})+1}\right) \\
& +\mathrm{d}\left(\mathrm{Ax}_{2 \mathrm{n}(\mathrm{k})+1}, \mathrm{Ax}_{2 \mathrm{~m}(\mathrm{k})}\right) \\
& \leq \mathrm{d}_{2 \mathrm{n}(\mathrm{k})}+\frac{1}{\mathrm{a}+\mathrm{c}} \phi\left\{\mathrm{d}\left(\mathrm{Sx}_{2 \mathrm{n}(\mathrm{k})+1}, \mathrm{Tx}_{2 \mathrm{~m}(\mathrm{k})}\right)\right. \text {, } \\
& \mathrm{d}\left(\mathrm{Sx}_{2 \mathrm{n}(\mathrm{k})+1}, \mathrm{Ax}_{2 \mathrm{n}(\mathrm{k})+1}\right), \mathrm{d}\left(\mathrm{Sx}_{2 \mathrm{n}(\mathrm{k})+1}, \mathrm{Ax}_{2 \mathrm{~m}(\mathrm{k})}\right) \text {, } \\
& \left.\mathrm{d}\left(\mathrm{Tx}_{2 \mathrm{~m}(\mathrm{k})}, \mathrm{Ax}_{2 \mathrm{n}(\mathrm{k})+1}\right), \mathrm{d}\left(\mathrm{Tx}_{2 \mathrm{~m}(\mathrm{k})}, \mathrm{Ax}_{2 \mathrm{~m}(\mathrm{k})}\right)\right\} \\
& +\frac{-b}{a+c} d\left(\mathrm{Ax}_{2 \mathrm{n}(\mathrm{k})}, \mathrm{Ax}_{2 \mathrm{~m}(\mathrm{k})-1}\right) \\
& \left(\frac{1}{\mathrm{a}+\mathrm{c}}\right) \phi\left\{\mathrm{d}\left(\mathrm{Ax}_{2 \mathrm{n}(\mathrm{k})}, \mathrm{Ax} \mathrm{x}_{2 \mathrm{~m}(\mathrm{k})-1}\right), \mathrm{d}\left(\mathrm{Ax}_{2 \mathrm{n}(\mathrm{k})}, \mathrm{Ax}_{2 \mathrm{n}(\mathrm{k})+1}\right)\right. \text {, } \\
& \mathrm{d}\left(\mathrm{Ax}_{2 \mathrm{n}(\mathrm{k})}, \mathrm{Ax}_{2 \mathrm{~m}(\mathrm{k})}\right), \mathrm{d}\left(\mathrm{Ax}_{2 \mathrm{~m}(\mathrm{k})-1}, \mathrm{Ax}_{2 \mathrm{n}(\mathrm{k})+1}\right) \text {, } \\
& \left.\mathrm{d}\left(\mathrm{Ax}_{2 \mathrm{~m}(\mathrm{k})-1}, \mathrm{Ax}_{2 \mathrm{~m}(\mathrm{k})}\right)\right\}+\left(\frac{-\mathrm{b}}{\mathrm{a}+\mathrm{c}}\right) \mathrm{d}\left(\mathrm{Ax}_{2 \mathrm{n}(\mathrm{k})}, \mathrm{Ax}_{2 \mathrm{~m}(\mathrm{k})-1}\right) \text {. }
\end{aligned}
$$

As $\mathrm{k} \rightarrow \infty$, we get

$$
\varepsilon \leq \frac{1}{\mathrm{a}+\mathrm{c}} \phi(\varepsilon, 0, \varepsilon, \varepsilon, 0)-\left(\frac{-\mathrm{b}}{\mathrm{a}+\mathrm{c}}\right) \varepsilon \leq\left(\frac{1-\mathrm{b}}{\mathrm{a}+\mathrm{c}}\right) \varepsilon<\varepsilon,
$$

a contradiction. Hence $\left\{\mathrm{Ax}_{\mathrm{n}}\right\}$ is a Cauchy sequence in $\mathrm{X}$ and so by completeness of $\mathrm{X},\left\{\mathrm{Ax}_{\mathrm{n}}\right\}$ converges to a point $\mathrm{z} \in$ $X$. Consequently the subsequences $\left\{\mathrm{Sx}_{2 \mathrm{n}+1}\right\}$ and $\left\{\mathrm{Tx}_{2 \mathrm{n}}\right\}$ of $\left\{\mathrm{Ax}_{\mathrm{n}}\right\}$ also converge to $\mathrm{z}$. Since $\{\mathrm{A}, \mathrm{S}\}$ is compatible, we get and therefore

$$
\lim _{n \rightarrow \infty}\left(\mathrm{ASx}_{2 \mathrm{n}+1}, \mathrm{SAx}_{2 \mathrm{n}+1}\right) \rightarrow 0
$$

$\lim _{n \rightarrow \infty} A_{S x_{2 n+1}} \lim _{n \rightarrow \infty} S_{A x} x_{2 n+1}=S\left(\lim _{n \rightarrow \infty} A x_{2 n+1}\right)=S z$.

Now we show that $\mathrm{Sz}=\mathrm{z}$. Suppose $\mathrm{Sz} \neq \mathrm{z}$, then from (ii) we have ,

$\operatorname{ad}\left(\mathrm{ASx}_{2 \mathrm{n}+1}, \mathrm{Ax}_{2 \mathrm{n}}\right)+\operatorname{bd}\left(\mathrm{S}^{2} \mathrm{x}_{2 \mathrm{n}+1}, \mathrm{Tx}_{2 \mathrm{n}}\right)+\mathrm{cd}\left(\mathrm{S}^{2} \mathrm{x}_{2 \mathrm{n}+1}, \mathrm{ASx}_{2 \mathrm{n}+1}\right)$ $\leq \phi\left\{d\left(S^{2} x_{2 n+1}, T x_{2 n}\right), d\left(A S x_{2 n+1}, S^{2} x_{2 n+1}\right), d\left(S^{2} x_{2 n+1}, A x_{2 n}\right)\right.$, $\left.\mathrm{d}\left(\mathrm{ASx}_{2 \mathrm{n}+1}, \mathrm{Tx}_{2 \mathrm{n}}\right), \mathrm{d}\left(\mathrm{Tx}_{2 \mathrm{n}}, \mathrm{Ax}_{2 \mathrm{n}}\right)\right\}$

In the limiting case, we get

$$
\begin{aligned}
& \mathrm{ad}(\mathrm{Sz}, \mathrm{z})+\mathrm{bd}(\mathrm{Sz}, \mathrm{z})+\mathrm{cd}(\mathrm{Sz}, \mathrm{z}) \leq \phi\{\mathrm{d}(\mathrm{Sz}, \mathrm{z}), \mathrm{d}(\mathrm{Sz}, \mathrm{Sz}), \\
& \mathrm{d}(\mathrm{Sz}, \mathrm{z}), \mathrm{d}(\mathrm{Sz}, \mathrm{z}), \mathrm{d}(\mathrm{z}, \mathrm{z})\} \\
& \text { or, }(\mathrm{a}+\mathrm{b}+\mathrm{c}) \mathrm{d}(\mathrm{Sz}, \mathrm{z}) \leq \phi\{\mathrm{d}(\mathrm{Sz}, \mathrm{z}), 0, \mathrm{~d}(\mathrm{Sz}, \mathrm{z}), \mathrm{d}(\mathrm{Sz}, \mathrm{z}), 0\} \\
& <\phi\{\mathrm{d}(\mathrm{Sz}, \mathrm{z}), \mathrm{d}(\mathrm{Sz}, \mathrm{z}), 2 \mathrm{~d}(\mathrm{Sz}, \mathrm{z}), \mathrm{d}(\mathrm{Sz}, \mathrm{z}), \mathrm{d}(\mathrm{Sz}, \mathrm{z})\} \\
& <\mathrm{d}(\mathrm{Sz}, \mathrm{z}) \text {, }
\end{aligned}
$$

a contradiction. Thus $\mathrm{z}$ is a fixed point of $\mathrm{S}$. Similarly taking $x=z$ and $y=x_{2 n}$ in (ii), we can show that $z$ is a fixed point of A. Lastly we claim that $\mathrm{z}$ is a fixed point of $\mathrm{T}$. Suppose it is not so, then

$$
\mathrm{d}(\mathrm{z}, \mathrm{Tz})>0 .
$$

Since $\mathrm{A}$ is compatible with $\mathrm{T}$ we have

and so

$$
\lim _{\mathrm{n} \rightarrow \infty} \mathrm{d}\left(\mathrm{ATx}_{2 \mathrm{n}}, \mathrm{TAx}_{2 \mathrm{n}}\right) \rightarrow 0
$$

$$
\lim _{n \rightarrow \infty} A x_{2 n}=\lim _{n \rightarrow \infty} \operatorname{TAx}_{2 n}=T\left(\lim _{n \rightarrow \infty} A x_{2 n}\right)=T z .
$$

Now from (ii), we have

$$
\operatorname{ad}\left(\mathrm{Az}, \mathrm{ATx}_{2 \mathrm{n}}\right)+\mathrm{bd}\left(\mathrm{Sz}, \mathrm{T}^{2} \mathrm{x}_{2 \mathrm{n}}\right)+\mathrm{cd}(\mathrm{Sz}, \mathrm{Az})
$$

$\leq \phi\left\{d\left(\mathrm{Sz}_{1} \mathrm{~T}^{2} \mathrm{x}_{2 \mathrm{n}}\right), \mathrm{d}(\mathrm{Sz}, \mathrm{Az}), \mathrm{d}\left(\mathrm{Sz}, \mathrm{ATx}_{2 \mathrm{n}}\right), \mathrm{d}\left(\mathrm{T}^{2} \mathrm{x}_{2 \mathrm{n}}, \mathrm{Az}\right)\right.$, $\left.\mathrm{d}\left(\mathrm{T}^{2} \mathrm{x}_{2 \mathrm{n}}, \mathrm{AT}_{2 \mathrm{n}}\right)\right\}$

As $n \rightarrow \infty$, we get

$$
\begin{aligned}
\mathrm{ad}(\mathrm{z}, \mathrm{Tz})+ & \mathrm{bd}(\mathrm{z}, \mathrm{Tz})+\mathrm{cd}(\mathrm{z}, \mathrm{z}) \\
& \leq \phi\{\mathrm{d}(\mathrm{z}, \mathrm{Tz}), \mathrm{d}(\mathrm{z}, \mathrm{z}), \mathrm{d}(\mathrm{z}, \mathrm{Tz}), \mathrm{d}(\mathrm{Tz}, \mathrm{z}), \mathrm{d}(\mathrm{Tz}, \mathrm{Tz})\} \\
& <\phi\{\mathrm{d}(\mathrm{z}, \mathrm{Tz}), \mathrm{d}(\mathrm{z}, \mathrm{Tz}), 2 \mathrm{~d}(\mathrm{z}, \mathrm{Tz}), \mathrm{d}(\mathrm{Tz}, \mathrm{z}), \mathrm{d}(\mathrm{z}, \mathrm{Tz})\} \\
& <\mathrm{d}(\mathrm{z}, \mathrm{Tz})
\end{aligned}
$$

or, $(\mathrm{a}+\mathrm{b}-1) \mathrm{d}(\mathrm{z}, \mathrm{Tz})<0$

a contradiction, since $\mathrm{a}+\mathrm{b}>1$. 
Therefore $\mathrm{Tz}=\mathrm{z}$. Thus $\mathrm{z}$ is a common fixed point of A,S and T. From (ii), we can easily show that the fixed point is unique. This completes the proof.

In that follows we give a common fixed point result for four self mappings satisfying a more general inequality condition. This in turn generalizes the fixed point result of Mukherjee[6].

\subsection{Lemma (Kang et.al.[5]) :}

Let $(\mathrm{X}, \mathrm{d})$ be a metric space, $\mathrm{K} \subset \mathrm{X}$ and $\mathrm{A}, \mathrm{S}: \mathrm{K} \rightarrow \mathrm{K}$ be compatible mappings. If $\left\{\mathrm{x}_{\mathrm{n}}\right\}$ is a sequence in $\mathrm{K}$ and $\lim _{n \rightarrow \infty} A x_{n}=\lim _{n \rightarrow \infty} S x_{n}=t$ for some $t \in K$, then $\lim _{\mathrm{n} \rightarrow \infty} \mathrm{ASx}_{\mathrm{n}}=\mathrm{St}$ if $\mathrm{S}$ is continuous.

\subsection{Theorem II}

Let $(\mathrm{X}, \mathrm{d})$ be a complete metric space and $\mathrm{A}, \mathrm{B}, \mathrm{S}$ and $\mathrm{T}$ be self mappings of $X$. Suppose that $S$ and $T$ are continuous. The mapping pairs $\{\mathrm{A}, \mathrm{S}\}$ and $\{\mathrm{B}, \mathrm{T}\}$ are compatible with $\mathrm{A}(\mathrm{X}) \subset \mathrm{T}(\mathrm{X})$ and $\mathrm{B}(\mathrm{X}) \subset \mathrm{S}(\mathrm{X})$ and there exists a upper semi continuous function $\phi:[0, \infty) \rightarrow[0, \infty)$, which is non decreasing and

$\phi(\mathrm{t})<\mathrm{t}$ for all $\mathrm{t}>0$. Let $\mathrm{A}, \mathrm{B}, \mathrm{S}$ and $\mathrm{T}$ satisfy the condition :

(vi) $\mathrm{d}(\mathrm{Sx}, \mathrm{Ty}) \leq \phi(\max \{\mathrm{d}(\mathrm{Ax}, \mathrm{By}), \mathrm{d}(\mathrm{Sx}, \mathrm{Ax}), \mathrm{d}(\mathrm{Ty}, \mathrm{By})$, $\mathrm{d}(\mathrm{Ty}, \mathrm{Bx}), \mathrm{d}(\mathrm{Sx}, \mathrm{By}), \mathrm{d}(\mathrm{Ty}, \mathrm{Ay})\})$

Then A, B, S and T have a unique common fixed point in $\mathrm{X}$.

Proof: Since $\mathrm{A}(\mathrm{X}) \subset \mathrm{T}(\mathrm{X})$ and $\mathrm{B}(\mathrm{X}) \subset \mathrm{S}(\mathrm{X})$, we can choose a sequence $\left\{\mathrm{x}_{\mathrm{n}}\right\}$ in $\mathrm{X}$ such that

$$
\mathrm{Sx}_{2 \mathrm{n}}=\mathrm{Bx}_{2 \mathrm{n}-1} \text { and } \mathrm{Tx}_{2 \mathrm{n}-1}=\mathrm{Ax}_{2 \mathrm{n}-2}
$$

for all $\mathrm{n}$ in the set $\mathrm{N}$ of all positive integers. Let

(vii) $\mathrm{y}_{2 \mathrm{n}-1}=\mathrm{Tx}_{2 \mathrm{n}-1}=\mathrm{Ax}_{2 \mathrm{n}-2}$ and $\mathrm{y}_{2 \mathrm{n}}=\mathrm{Sx}_{2 \mathrm{n}}=\mathrm{Bx}_{2 \mathrm{n}-1}$ for $\mathrm{n} \in \mathrm{N}$ Now from (vi), we have

$$
\begin{gathered}
\mathrm{d}\left(\mathrm{Sx}_{2 \mathrm{n}}, \mathrm{Tx}_{2 \mathrm{n}-1}\right) \leq \\
\mathrm{d}\left(\mathrm{Tx}_{2 \mathrm{n}-1}, \mathrm{Bx}_{2 \mathrm{n}-1}\right), \mathrm{d}\left(\mathrm{Tx}_{2 \mathrm{n}-1}, \mathrm{Bx}_{2 \mathrm{n}}\right), \mathrm{d}\left(\mathrm{Sx}_{2 \mathrm{n}}, \mathrm{Bx}_{2 \mathrm{n}-1}\right), \\
\left.\left.\mathrm{d}\left(\mathrm{Tx}_{2 \mathrm{n}-1}, \mathrm{Ax}_{2 \mathrm{n}-1}\right)\right\}\right)
\end{gathered}
$$

or, $\mathrm{d}\left(\mathrm{y}_{2 \mathrm{n}}, \mathrm{y}_{2 \mathrm{n}-1}\right) \leq \phi\left(\max \left\{\mathrm{d}\left(\mathrm{y}_{2 \mathrm{n}+1}, \mathrm{y}_{2 \mathrm{n}}\right), \mathrm{d}\left(\mathrm{y}_{2 \mathrm{n}}, \mathrm{y}_{2 \mathrm{n}+1}\right)\right.\right.$,

$$
\left.\left.\mathrm{d}\left(\mathrm{y}_{2 \mathrm{n}-1}, \mathrm{y}_{2 \mathrm{n}}\right), \mathrm{d}\left(\mathrm{y}_{2 \mathrm{n}-1}, \mathrm{y}_{2 \mathrm{n}-1}\right), \mathrm{d}\left(\mathrm{y}_{2 \mathrm{n}}, \mathrm{y}_{2 \mathrm{n}}\right), \mathrm{d}\left(\mathrm{y}_{2 \mathrm{n}-1}, \mathrm{y}_{2 \mathrm{n}}\right)\right\}\right) \text {. }
$$

Let $d_{2 n}=d\left(y_{2 n}, y_{2 n+1}\right)>0$ for all $n=0,1,2, \ldots$. Then we get

$$
\mathrm{d}_{2 \mathrm{n}-1} \leq \phi\left(\max \left\{\mathrm{d}_{2 \mathrm{n}}, \mathrm{d}_{2 \mathrm{n}}, \mathrm{d}_{2 \mathrm{n}-1}, 0,0, \mathrm{~d}_{2 \mathrm{n}-1}\right\}\right)=\phi\left(\mathrm{d}_{2 \mathrm{n}}\right)<\mathrm{d}_{2 \mathrm{n}} .
$$

Hence $\mathrm{d}_{2 \mathrm{n}-1}<\mathrm{d}_{2 \mathrm{n}}$. Similarly we can show that $\mathrm{d}_{2 \mathrm{n}}<\mathrm{d}_{2 \mathrm{n}+1}$.

Therefore $\left\{\mathrm{d}_{\mathrm{n}}\right\}$ is non decreasing sequence in $\mathrm{R}$. We now show that $\left\{\mathrm{y}_{\mathrm{n}}\right\}$ is a Cauchy sequence. Suppose that it is not so, then there exists an $\varepsilon>0$ such that for any integer $2 \mathrm{k}, \mathrm{k}=$ $0,1,2, \ldots$ there exist two sequences $\{2 \mathrm{~m}(\mathrm{k})\}_{\mathrm{k}}$ and $\{2 \mathrm{n}(\mathrm{k})\}_{\mathrm{k}}$, $\mathrm{k} \in \mathrm{N}$ with $2 \mathrm{k}<2 \mathrm{n}(\mathrm{k})<2 \mathrm{~m}(\mathrm{k})$, for which

(viii) $\mathrm{d}\left(\mathrm{y}_{2 \mathrm{n}(\mathrm{k})}, \mathrm{y}_{2 \mathrm{~m}(\mathrm{k})}\right)>\varepsilon$

If $2 \mathrm{~m}(\mathrm{k})$ denote the smallest integer exceeding $2 \mathrm{n}(\mathrm{k})$ satisfying (viii), we have

(ix) $\mathrm{d}\left(\mathrm{y}_{2 \mathrm{n}(\mathrm{k})}, \mathrm{y}_{2 \mathrm{~m}(\mathrm{k})-2}\right) \leq \varepsilon$

Then,

$\left.\varepsilon<\mathrm{d}\left(\mathrm{y}_{2 \mathrm{n}(\mathrm{k})}, \mathrm{y}_{2 \mathrm{~m}(\mathrm{k})}\right)\right) \leq \mathrm{d}\left(\mathrm{y}_{2 \mathrm{n}(\mathrm{k})}, \mathrm{y}_{2 \mathrm{~m}(\mathrm{k})-2}\right)+\mathrm{d}_{2 \mathrm{~m}(\mathrm{k})-2}+\mathrm{d}_{2 \mathrm{~m}(\mathrm{k})-1}$.

So by (ix) and as $\mathrm{k} \rightarrow \infty$, we get

(x) $\lim _{\mathrm{k} \rightarrow \infty} \mathrm{d}\left(\mathrm{y}_{2 \mathrm{n}(\mathrm{k})}, \mathrm{y}_{2 \mathrm{~m}(\mathrm{k})}\right)=\varepsilon$

Now using $(\mathrm{x})$ in the triangle inequalities

$\left|\mathrm{d}\left(\mathrm{y}_{2 \mathrm{~m}(\mathrm{k})}, \mathrm{y}_{2 \mathrm{n}(\mathrm{k})+2}\right)-\mathrm{d}\left(\mathrm{y}_{2 \mathrm{~m}(\mathrm{k})+1}, \mathrm{y}_{2 \mathrm{n}(\mathrm{k})+1}\right)\right| \leq \mathrm{d}_{2 \mathrm{~m}(\mathrm{k})}+\mathrm{d}_{2 \mathrm{n}(\mathrm{k})+1}$

$\left|\mathrm{d}\left(\mathrm{y}_{2 \mathrm{n}(\mathrm{k})+1}, \mathrm{y}_{2 \mathrm{~m}(\mathrm{k})+1}\right)-\mathrm{d}\left(\mathrm{y}_{2 \mathrm{n}(\mathrm{k})}, \mathrm{y}_{2 \mathrm{~m}(\mathrm{k})}\right)\right| \leq \mathrm{d}_{2 \mathrm{~m}(\mathrm{k})}+\mathrm{d}_{2 \mathrm{n}(\mathrm{k})}$ and

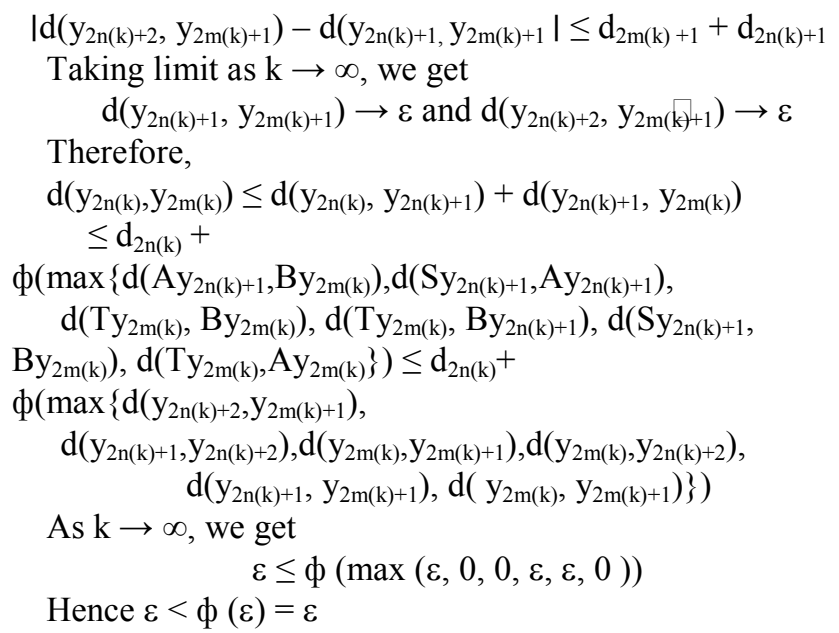

a contradiction. Hence $\left\{\mathrm{y}_{\mathrm{n}}\right\}$ is a Cauchy sequence in $\mathrm{X}$. Suppose $\mathrm{y}_{\mathrm{n}} \rightarrow \mathrm{y} \in X$, then the subsequences $\left\{\mathrm{y}_{2 \mathrm{n}}\right\}$ and $\left\{\mathrm{y}_{2 \mathrm{n}-1}\right\}$ also converge to y. Since $\{\mathrm{A}, \mathrm{S}\}$ and $\{\mathrm{B}, \mathrm{T}\}$ are both compatible, it follows from the continuity of $\mathrm{S}$ and $\mathrm{T}$ that

(xi) $\mathrm{Ty}_{2 \mathrm{n}-1} \rightarrow \mathrm{Ty}, \mathrm{By}_{2 \mathrm{n}-1} \rightarrow \mathrm{Ty}, \mathrm{Sy}_{2 \mathrm{n}} \rightarrow \mathrm{Sy}, \mathrm{Ay}_{2 \mathrm{n}} \rightarrow \mathrm{Sy}$

Again from (vi), we get

$$
\begin{aligned}
\mathrm{d}\left(\mathrm{Sy}_{2 \mathrm{n}}, \mathrm{Ty}_{2 \mathrm{n}-1}\right) \leq & \phi\left(\operatorname { m a x } \left\{\mathrm{d}\left(\mathrm{Ay}_{2 \mathrm{n}}, \mathrm{By} \mathrm{y}_{2 \mathrm{n}-1}\right), \mathrm{d}\left(\mathrm{Sy}_{2 \mathrm{n}}, \mathrm{Ay}_{2 \mathrm{n}}\right),\right.\right. \\
\mathrm{d}\left(\mathrm{Ty}_{2 \mathrm{n}-1}, \mathrm{By}_{2 \mathrm{n}-1}\right), \mathrm{d}\left(\mathrm{Ty}_{2 \mathrm{n}-1}, \mathrm{By}_{2 \mathrm{n}}\right), \mathrm{d}\left(\mathrm{Sy}_{2 \mathrm{n}}, \mathrm{By}_{2 \mathrm{n}-1}\right), & \left.\left.\mathrm{d}\left(\mathrm{Ty}_{2 \mathrm{n}-1}, \mathrm{Ay}_{2 \mathrm{n}-1}\right)\right\}\right) .
\end{aligned}
$$

By the upper semi continuity of $\phi$ and (xi), we get

$\mathrm{d}($ Sy, Ty $) \leq \phi(\max \{\mathrm{d}(\mathrm{Sy}, \mathrm{Ty}), \mathrm{d}(\mathrm{Sy}, \mathrm{Sy}), \mathrm{d}(\mathrm{Ty}, \mathrm{Ty})$,

d(Ty,Ty), d(Ty,Ty), d(Sy,Ty), d(Ty,Sy) $\})$

$\leq \phi(\max \{\mathrm{d}(\mathrm{Sy}, \mathrm{Ty}), 0,0,0,0, \mathrm{~d}(\mathrm{Sy}, \mathrm{Ty}), \mathrm{d}(\mathrm{Sy}, \mathrm{Ty})\})$

$\leq \phi(\mathrm{d}(\mathrm{Sy}, \mathrm{Ty}))<\mathrm{d}(\mathrm{Sy}, \mathrm{Ty})$.

This implies $\mathrm{Sy}=\mathrm{Ty}$. Similarly we can obtain that

(xii) $\mathrm{Sy}=\mathrm{By}, \mathrm{Ty}=\mathrm{Ay}$ and so, $\mathrm{Ay}=\mathrm{By}=\mathrm{Sy}=\mathrm{Ty}$

Again from (vi), we have

$\mathrm{d}\left(\mathrm{Sx}_{2 \mathrm{n}}, \mathrm{Ty}\right) \leq \phi\left(\max \left\{\mathrm{d}\left(\mathrm{Ax}_{2 \mathrm{n}}, \mathrm{By}\right), \mathrm{d}\left(\mathrm{Sx}_{2 \mathrm{n}}, \mathrm{Ax} x_{2 \mathrm{n}}\right), \mathrm{d}(\mathrm{Ty}, \mathrm{By})\right.\right.$, $\left.\left.\mathrm{d}\left(\mathrm{Ty}, \mathrm{Bx}_{2 \mathrm{n}}\right), \mathrm{d}\left(\mathrm{Sx}_{2 \mathrm{n}}, \mathrm{By}\right), \mathrm{d}(\mathrm{Ty}, \mathrm{Ay})\right\}\right)$

$\leq \phi(\max \{d(y, T y), d(y, y), d(T y, T y), d(T y, y)$, $\mathrm{d}(\mathrm{y}, \mathrm{Ty}), \mathrm{d}(\mathrm{Ty}, \mathrm{Ty})\})$

or, $\mathrm{d}(\mathrm{y}, \mathrm{Ty}) \leq \phi(\max \{\mathrm{d}(\mathrm{y}, \mathrm{Ty}), 0,0, \mathrm{~d}(\mathrm{Ty}, \mathrm{y}), \mathrm{d}(\mathrm{y}, \mathrm{Ty}), 0\})$ $\leq \phi(\mathrm{d}(\mathrm{y}, \mathrm{Ty}))<\mathrm{d}(\mathrm{y}, \mathrm{Ty})$.

Therefore $\mathrm{y}=\mathrm{Ty}$. Hence $\mathrm{y}=\mathrm{Ty}=\mathrm{Ay}=\mathrm{By}=\mathrm{Sy}$. Thus $\mathrm{y}$ is the common fixed point of $\mathrm{A}, \mathrm{B}, \mathrm{S}$ and $\mathrm{T}$.

To prove uniqueness let $\mathrm{x}(\neq \mathrm{y})$ be another common fixed point of $A, B, S$ and $T$. Then from (vi), we get

$$
\begin{gathered}
d(\text { Sx,Ty }) \leq \phi(\max \{d(A x, B y), d(\text { Sx,Ax }), d(T y, B y), \\
d(T y, B x), d(S x, B y), d(T y, A y)\}) \\
\text { or, } d(x, y) \leq \phi(\max \{d(x, y), d(x, x), d(y, y), d(y, x), \\
d(x, y), d(y, y)\}) \\
\text { or, } d(x, y) \leq \phi(d(x, y))<d(x, y)
\end{gathered}
$$

and so, we get $\mathrm{x}=\mathrm{y}$. Thus $\mathrm{A}, \mathrm{B}, \mathrm{S}$ and $\mathrm{T}$ have a unique common fixed point $\mathrm{y}$ in $\mathrm{X}$.

\section{Conclusions}

The inequality conditions (ii) and (vi) of our respective results are more general by involving a $\phi$ function than the conditions (1), (2) and (3) of the results of Som[10], Taskovic[12] and that of Mukherjee[6]. Moreover the nature 
of the functions are more restricted due to compatibility and we get common fixed points for such mappings.

\section{REFERENCES}

[1] Fisher, B. and Sessa, S., Common fixed points of weakly commuting mappings, Bull. Acad. Polon Sci.Ser.Sci.Math. 35(1987), 341-349

[2] Hadzic, O., Common fixed point theorem for family of mappings in complete metricspaces, Math. Japon, 29(1984), 127-134

[3] Jungck, G., Commuting mappings and fixed points, Amer. Math. Monthly, 83(1976),261- 263

[4] Compatible mappings and common fixed points, Int.J.Math. \&Math.Sci. 9(1986), 771-779

[5] Kang, S. M., Cho, Y. J., Jungck, G., Common fixed points of compatible mappings, Int. J. Math. \&Math. Sci.13(1990),
$61-65$

[6] Mukherjee, R. N., Compatible mappings and fixed points, Ind.J.Pure Appl.Math. 12(8) (1981), 930

[7] Pathak, H. K., Weak commuting mappings and fixed points, Ind.J.Pure Appl. Math. 17(2)( 1986), 201-211

[8] Sessa, S., On a weak commutativity condition in fixed point considerations, Publ.Inst. Math. 32(46) (1982 ), 149-153

[9] Sessa, S., Mukerjee, R. N. \& Som, T., A common fixed point theorem for weakly commuting mappings, Math. Japonica 31(2) (1986), 235-245

[10] Som, T., Some fixed point theorems on metric and Banach spaces, Ind. Jour. Pure Appl. Math. 16(6) (1985),575-585

[11] Singh, S. P. \& Meade, B. A., On common fixed point theorem, Bull.Aus. Math. Soc.16 (1977),49-53

[12] Taskovic, M. R., Some results in fixed point theory, Publ. Inst. Math. 20(34) ( 1976), 231- 242

[13] Yeh, C. C., On common fixed points of continuous mappings, Ind. J. Appl. Math.10(1979), 415-420 